\title{
Risk of deep venous thrombosis in elective neurosurgical procedures: a prospective, Doppler ultrasound-based study in children 12 years of age or younger
}

\author{
Andrea G. Scherer, MD, lan K. White, MD, Kashif A. Shaikh, MD, Jodi L. Smith, PhD, MD, \\ Laurie L. Ackerman, MD, and Daniel H. Fulkerson, MD
}

Department of Neurological Surgery, Division of Pediatric Neurosurgery, Goodman Campbell Brain and Spine, Indiana University School of Medicine, Indianapolis, Indiana

OBJECTIVE The risk of venous thromboembolism (VTE) from deep venous thrombosis (DVT) is significant in neurosurgical patients. VTE is considered a leading cause of preventable hospital deaths and preventing DVT is a closely monitored quality metric, often tied to accreditation, hospital ratings, and reimbursement. Adult protocols include prophylaxis with anticoagulant medications. Children's hospitals may adopt adult protocols, although the incidence of DVT and the risk or efficacy of treatment is not well defined. The incidence of DVT in children is likely less than in adults, although there is very little prospectively collected information. Most consider the risk of DVT to be extremely low in children 12 years of age or younger. However, this consideration is based on tradition and retrospective reviews of trauma databases. In this study, the authors prospectively evaluated pediatric patients undergoing a variety of elective neurosurgical procedures and performed Doppler ultrasound studies before and after surgery.

METHODS A total of 100 patients were prospectively enrolled in this study. All of the patients were between the ages of 1 month and 12 years and were undergoing elective neurosurgical procedures. The 91 patients who completed the protocol received a bilateral lower-extremity Doppler ultrasound examination within 48 hours prior to surgery. Patients did not receive either medical or mechanical DVT prophylaxis during or after surgery. The ultrasound examination was repeated within 72 hours after surgery. An independent, board-certified radiologist evaluated all sonograms. We prospectively collected data, including potential risk factors, details of surgery, and details of the clinical course. All patients were followed clinically for at least 1 year.

RESULTS There was no clinical or ultrasound evidence of DVT or VTE in any of the 91 patients. There was no clinical evidence of VTE in the 9 patients who did not complete the protocol.

CONCLUSIONS In this prospective study, no DVTs were found in 91 patients evaluated by ultrasound and 9 patients followed clinically. While the study is underpowered to give a definitive incidence, the data suggest that the risk of DVT and VTE is very low in children undergoing elective neurosurgical procedures. Prophylactic protocols designed for adults may not apply to pediatric patients.

Clinical trial registration no.: NCT02037607 (clinicaltrials.gov)

https://thejns.org/doi/abs/10.3171/2017.3.PEDS16588

KEY WORDS venous thromboembolism; deep venous thrombosis; pediatric; neurosurgery; elective; ultrasound; vascular disorders

$\Delta$ DULT neurosurgical patients have a significant risk for developing venous thromboembolism (VTE), including both deep venous thrombosis (DVT) and pulmonary embolism (PE), ${ }^{1,25}$ VTE is considered the leading cause of preventable hospital deaths. ${ }^{5}$ Prevention of DVT is an increasingly prevalent "quality metric" used to rank hospitals and potentially determine reimburse- ment. Multiple national groups, including the Centers for Medicare and Medicaid, Joint Commission, and Leapfrog Group, recommend and track compliance with protocols. Protocols include medical anticoagulation; while this has been shown to reduce the incidence of DVT, it may increase the risk of bleeding complications. ${ }^{1,24}$

There is a substantial amount of literature regarding the

ABBREVIATIONS BMI = body mass index; DVT = deep venous thrombosis; $\mathrm{HIT}=$ heparin-induced thrombocytopenia; $I$ RB $=$ institutional review board; $\mathrm{LMWH}=$ lowmolecular-weight heparin; $\mathrm{OR}=$ operating room; $\mathrm{PE}=$ pulmonary embolism; VTE = venous thromboembolism.

SUBMITTED October 19, 2016. ACCEPTED March 10, 2017.

INCLUDE WHEN CITING Published online May 5, 2017; DOI: 10.3171/2017.3.PEDS16588. 
risk, incidence, and treatment of VTE in adults. However, data on VTE in children are sparse. Much of the available pediatric data comes from retrospective database reviews and trauma registries. Pediatric hospitals are often charged with developing DVT prophylaxis protocols, despite a lack of prospective data. A survey report of children's hospitals showed that protocols varied widely, with some hospitals simply adopting or modifying adult procedures. ${ }^{8}$

Protocols based on adult data may not be sensible in children. The limited available data suggest that the risk of DVT is much lower in children. ${ }^{43}$ In 1994, the estimated incidence of DVT in children from the Canadian Childhood Thrombophilia registry was 0.07 per 10,000 children; this was less than the estimated incidence of 5.6-16 per 10,000 adults...$^{34,45,46}$ Based on trauma data, the relative risk of DVT in children is 7.2 times less than in adults, or 24/10,000 pediatric trauma patients. ${ }^{8,23}$ Patients less than 12 years of age are considered to have a very low risk of developing DVT. ${ }^{27}$ However, this consideration is based on either retrospective trauma data or tradition.

Pharmacological prophylaxis is generally considered safe in adults, but the risks are not well established in children. Low-molecular-weight heparins (LMWHs) are commonly used for surgical prophylaxis. The appropriate dosing in children varies with the age and size of the patient and thus may be more difficult to manage than in adults. ${ }^{15}$ Heparin may theoretically cause heparin-induced thrombocytopenia (HIT), even with minimal doses. ${ }^{33,40}$ There is a small but definite risk of hemorrhagic complications with anticoagulants. ${ }^{36,42}$ Administering prophylactic medication to children based on adult protocols may subject neurosurgical patients to an unnecessary risk.

In this study, we attempted to evaluate the risk of developing DVT or subsequent VTE in pediatric neurosurgical patients aged 1 month to 12 years. We prospectively enrolled 100 consecutive patients scheduled for elective surgeries. Patients were evaluated with Doppler ultrasonography before and after surgery. Patients underwent surgery without either mechanical or pharmacological VTE prophylaxis. All data were collected prospectively; patients were followed clinically for at least 1 year.

\section{Methods}

The study began after approval from the Indiana University institutional review board (IRB). We received funding for the imaging of 100 patients. Patients were enrolled prospectively. Informed consent and assent were obtained per the guidelines of the Indiana University IRB. We enrolled 100 consecutive patients between the ages of 1 month to 12 years over the time period from 2013 through 2015. All patients were scheduled for an elective neurosurgical procedure performed under general anesthesia. Patients with a preexisting VTE, pregnancy, or known hypercoagulable state were excluded; all other patients were enrolled consecutively.

Patients enrolled in the study underwent a preoperative bilateral lower-extremity Doppler ultrasound examination within 48 hours of the surgery. A postoperative, bilateral lower-extremity study was performed within 72 hours after the surgery. Patients did not receive medical or mechanical VTE prophylaxis during surgery or in the immediate postoperative period. An independent, boardcertified radiologist evaluated all of the sonograms.

Demographic information, radiological results, and clinical outcomes were prospectively collected. Data points included age at time of the procedure, sex, diagnosis, comorbidities, body mass index (BMI) for children over 2 years of age, presence of a central line (jugular, subclavian, or femoral), duration of surgery, type of surgery, position during surgery (supine or prone), ultrasonography results, adverse events, and clinical outcomes. All patients were clinically followed for at least 1 year after the surgery.

This study was registered with the ClinicalTrials.gov database (http://clinicaltrials.gov), and its registration no. is NCT00622778.

\section{Results}

One hundred patients were initially enrolled in the study. Nine patients withdrew, either due to patient preference or deviation from the protocol. Of the 9 patients who withdrew, 2 did not have postoperative ultrasound studies ordered; 7 were unable to hold still for either the preoperative or postoperative study. All 9 patients were followed clinically after surgery; none had clinical evidence of VTE.

The study group thus consisted of 91 patients. The demographic data are shown in tabular form in Table 1 and in graph form in Fig. 1. The ages ranged from 1 month to just under 12 years. The median age was 5.95 years. There was a predominance of spine surgical procedures, although a variety of cases were represented. The most common procedure was tethered spinal cord release $(\mathrm{n}=$ $39)$, followed by Chiari decompression $(\mathrm{n}=17)$ and complex (instrumented) spine surgery $(n=13)$.

The surgical details are shown in Table 2 . The majority $(80.2 \%)$ of patients were placed in the prone position. The total time in the operating room (OR) averaged 3:42:44 $\pm 1: 37: 51$. Figure 2 shows bar graphs of the surgical time and the total time in the OR. There was only 1 patient with a venous central line. There were 3 complications in the perioperative period -1 patient had a postoperative CSF leak after a tethered cord release, 1 patient had feeding dysfunction prolonging his hospital stay, and 1 patient had asymptomatic premature ventricular contractions requiring extended cardiac monitoring. No patients had either clinical or ultrasound evidence of DVT. All patients were followed for at least 1 year. No patient developed clinical evidence of VTE in the postoperative period.

\section{Discussion}

Venous thromboembolism affects an estimated $300,000-600,000$ people in the United States each year. ${ }^{5}$ The financial burden is significant, with a total estimated cost of $\$ 2$ billion to $\$ 10$ billion per year. The clinical consequences may be devastating. Up to $30 \%$ of all adult patients with DVT die within 30 days of diagnosis. VTE is considered the leading cause of preventable hospital death. $^{5}$

Neurosurgical patients are at particular risk of developing DVT and VTE. The estimated incidence of VTE 
TABLE 1. Demographic and clinical characteristics of the 91 patients in the study group

\begin{tabular}{lc}
\hline \multicolumn{1}{c}{ Characteristic } & Value \\
\hline Sex & \\
\hline Male & $44(48.4 \%)$ \\
\hline Female & $47(51.6 \%)$ \\
\hline Age in yrs & $5.4 \pm 9.2$ \\
\hline Mean \pm SD & 5.95 \\
\hline Median & $4(4.4 \%)$ \\
\hline History of cancer & \\
\hline BMl (age $>2$ yrs) in kg/m ${ }^{2}$ & $18.02 \pm 4.21$ \\
\hline Mean \pm SD & 16.6 \\
\hline Median & $39(42.9 \%)$ \\
\hline Surgical procedure & $4(4.4 \%)$ \\
\hline Tethered cord release & $13(14.3 \%)$ \\
\hline Brain tumor & $17(18.7 \%)$ \\
\hline Complex spine & $3(3.3 \%)$ \\
\hline Chiari decompression & $2(2.2 \%)$ \\
\hline Seizure surgery & $3(3.3 \%)$ \\
\hline Baclofen pump/spasticity & $5(5.5 \%)$ \\
\hline Cranial reconstruction & $5(5.5 \%)$ \\
\hline Complex hydrocephalus &
\end{tabular}

in untreated adult neurosurgical patients is $22 \%-45 \% .^{16}$ Risk factors are well established for adults and include advanced age, history of previous VTE, trauma, surgery, hospitalization and ICU care, cancer, paralysis, obesity, venous catheterization, pregnancy, estrogen use, and a variety of predisposing medical conditions (thrombophilias, myocardial infarction, systemic lupus erythematosus, and multiple others). ${ }^{19,29,30,41}$ Risk factors in children are less defined. In retrospective studies, risk factors for DVT in children include central venous lines, obesity, mechanical ventilation, preexisting coagulopathies, transfusion,

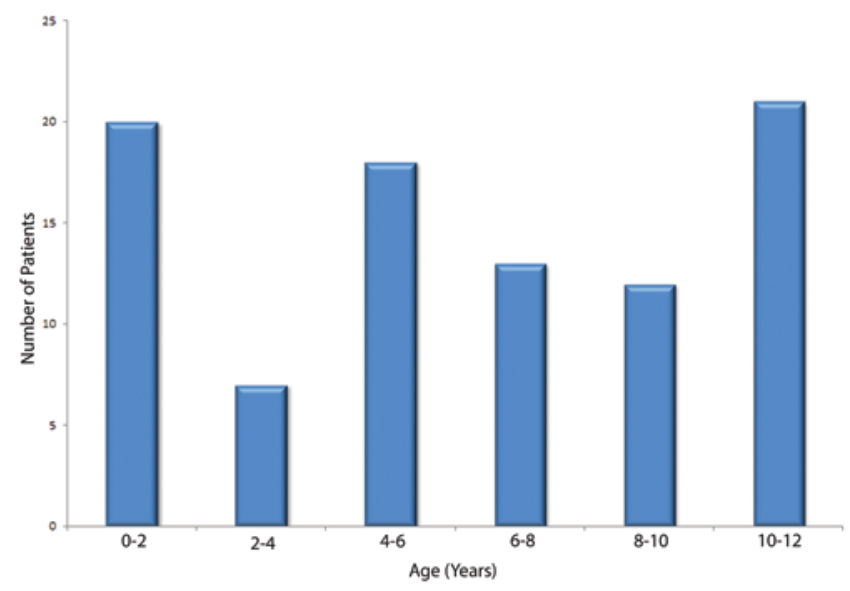

FIG. 1. Bar graph showing the age distribution of the study population. The $x$-axis intervals include the right boundary and exclude the left. Figure is available in color online only.
TABLE 2. Surgical and clinical data of the study group $(n=91)$

\begin{tabular}{|c|c|}
\hline Variable & Value \\
\hline \multicolumn{2}{|l|}{ Surgical position } \\
\hline Supine & $18(19.8 \%)$ \\
\hline Prone & $73(80.2 \%)$ \\
\hline \multicolumn{2}{|l|}{ Duration, mean $\pm S D$} \\
\hline Time under anesthesia & $3: 42: 44 \pm 1: 37: 51$ \\
\hline Op time & $2: 19: 59 \pm 1: 24: 13$ \\
\hline \multicolumn{2}{|l|}{ Central line } \\
\hline Jugular & 0 \\
\hline Subclavian & 0 \\
\hline Femoral & $1(1.1 \%)$ \\
\hline \multicolumn{2}{|l|}{ Postop risk factors } \\
\hline Bed rest for $24 \mathrm{hrs}$ & $33(36.3 \%)$ \\
\hline ICU care & $32(35.2 \%)$ \\
\hline Medical/surgical complications & $3(3.3 \%)^{*}$ \\
\hline DVT on preop ultrasound & 0 \\
\hline DVT on postop ultrasound & 0 \\
\hline Clinical DVT/VTE in follow-up period & 0 \\
\hline CSF leak & $1(1.1 \%)$ \\
\hline Postop feeding dysfunction & $1(1.1 \%)$ \\
\hline Asymptomatic PVCs & $1(1.1 \%)$ \\
\hline
\end{tabular}

$\mathrm{ICU}=$ intensive care unit; PVCs = premature ventricular contractions.

higher Injury Severity Score, sepsis, trauma, malignancy, orthopedic surgery, and cranial surgical procedures. ${ }^{2,12,27}$, $28,34,49$ The authors of a previous ultrasound study in primarily adult neurosurgical patients noted that the majority of perioperative DVTs occurred within 1 week of surgery and that the risk correlated with the duration of surgery. ${ }^{32}$

A growing body of literature demonstrates a reduction of VTE in neurosurgical patients with pharmacological prophylaxis.,11,21 Prevention protocols are becoming an increasingly measured "quality metric" for hospitals. The Surgical Care Improvement Project (SCIP) was implemented in 2006 by the Centers for Medicare and Medicaid in conjunction with the Joint Commission and the Leapfrog Group. One core goal of the SCIP initiative is to prevent VTE. Increasingly, reimbursement for services is linked to quality metrics. While this is now accepted in adult practice, there is little direction in children's hospitals. A survey report of children's hospitals documented a wide disparity in policy and also noted that some pediatric hospitals simply adopted a modified version of the adult protocol. ${ }^{8}$

While the incidence of VTE has been thoroughly studied in adults, the available data on pediatric VTE are sparse. What data exist suggest that the risk of DVT and VTE is much lower in children. In large retrospective database analyses, VTE occurred in $0.2 \%-1 \%$ of pediatric trauma patients, compared with $20 \%-58 \%$ of adult trauma patients. ${ }^{10,12,13,47,48}$ There is a prevailing opinion that children under 12 are at extremely low risk for DVT based on the trauma literature and tradition. ${ }^{27}$ However, there is very little scientific study to support this opinion. Van Arendonk et al. examined the data on 402,329 patients registered in the United States National Trauma Data Bank. 

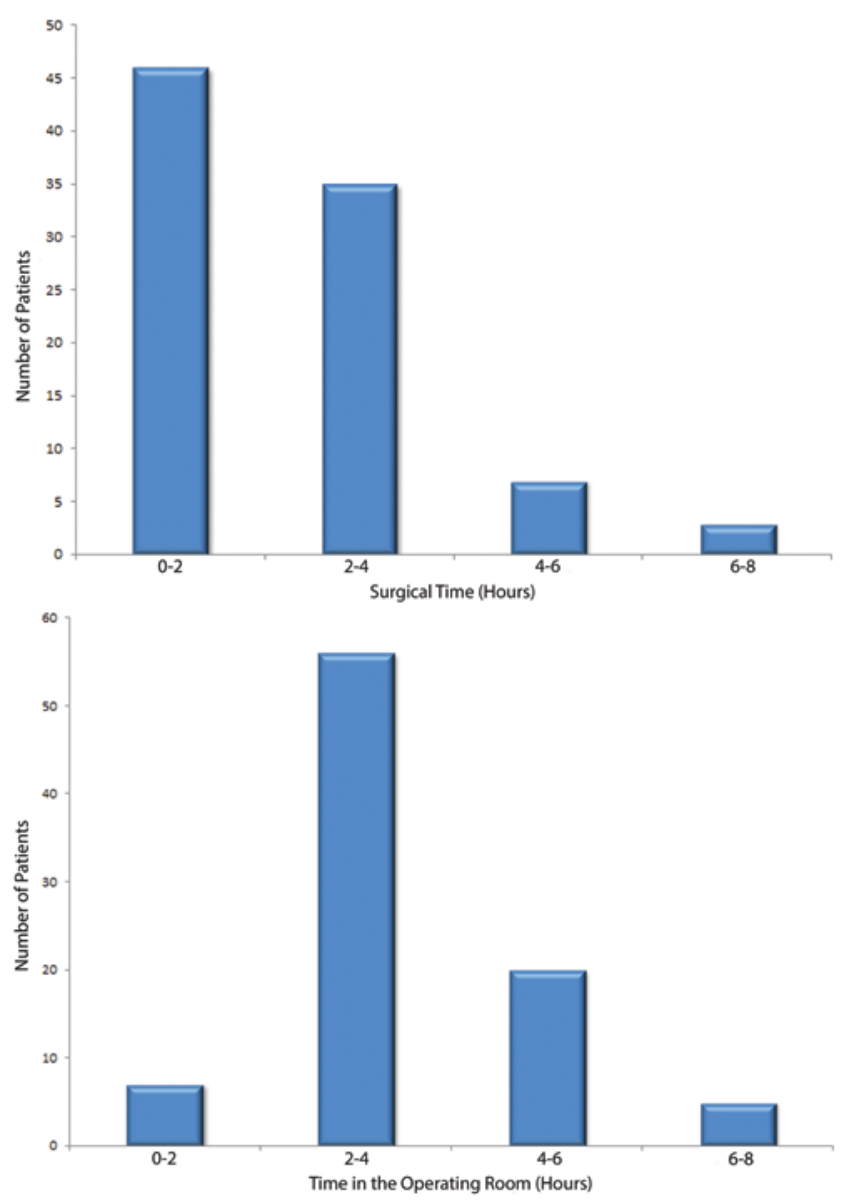

FIG. 2. Bar graphs of the surgical time (upper) and total time in the OR (lower) for the study population. Figure is available in color online only.

The incidence of VTE in children less than 12 years of age was $0 \% .^{47}$ While the data strongly suggest that the incidence of VTE is much lower in children, extrapolating policy for elective neurosurgical procedures from trauma data may be problematic.

There is evidence that the overall incidence of DVT and VTE in children is increasing, likely from a combination of improved diagnostics and increased survival of children with severe medical issues. ${ }^{20,39}$ In addition, children may tolerate VTE much better than adults. In general pediatrics, the mortality rate following the diagnosis of PE ranges from $1 \%$ to $8 \%$, much less than the $30 \%$ cited in adults. ${ }^{37,39,44}$ In a retrospective review of an orthopedic database of 14,776 pediatric surgical patients, 15 patients were diagnosed with VTE. While the hospitalization of these patients was prolonged, none died within 30 days. ${ }^{4}$ Others speculate that children tolerate PE better than adults because of fewer cardiopulmonary comorbidities and greater pulmonary reserve. ${ }^{6}$ Children may develop a DVT without classic clinical manifestations of calf swelling, redness, tenderness, or Homan's sign..$^{14}$ A retrospective study based purely on clinical diagnosis codes may therefore underestimate the true incidence of VTE. One of our goals in this project was to evaluate whether our patients developed asymptomatic DVT.
There are very few prospective studies evaluating the incidence or risk of VTE in neurosurgical patients. One prospective ultrasound study in adult neurosurgical patients found an incidence of $5.6 \%$. Interestingly, $81 \%$ of the DVTs diagnosed by ultrasound were asymptomatic at the time of discovery. ${ }^{17}$ There are very few prospective studies in children and none that focus on those less than 12 years of age outside of the trauma literature. Rohrer et al. ${ }^{43}$ prospectively evaluated 59 hospitalized pediatric patients with 2 or more risk factors for DVT using duplex ultrasound. They identified 1 DVT in a 17-year-old trauma patient. One of the only prospective studies evaluating elective surgical procedures was performed by Kaabachi et al. ${ }^{31}$ They evaluated 40 teenagers undergoing elective scoliosis surgery with screening ultrasound. Eight patients with known hematological hypercoagulable states were excluded. They found 2 children with asymptomatic small clots and no children with symptomatic thrombosis. All patients were older than 12 years.

LMWHs are often employed for surgical prophylaxis. Advantages of LMWHs include predictable pharmacokinetics, ease of administration, low cost (the hospital purchase price of a 40-mg enoxaparin dose at Riley Hospital for Children is \$3.68), low risk of HIT, and potentially fewer hemorrhagic complications compared with other anticoagulants. ${ }^{3,15}$ These considerations are based on adult studies. In children, the clearance of LMWH is age dependent, with infants requiring increased doses compared with older children. ${ }^{35}$ Children may require twicedaily dosing, as opposed to the usual once-daily dosing in adults. ${ }^{38}$ These factors may complicate the ease of administration cited in adults. Patients receiving any type of heparin have a risk of developing HIT, which may lead to devastating consequences; the risk is likely small, but it is poorly defined in children., ${ }^{9,33}$ There is also a small, but definite risk of hemorrhage with anticoagulants. ${ }^{7,26} \mathrm{In}$ a study retrospectively reviewing the use of LMWHs in pediatric neurosurgical patients, the authors noted a symptomatic bleeding rate of approximately $10 \%$ with one patient requiring a craniotomy for hematoma evacuation..$^{22}$ Other studies have found the risk of major bleeding in children to be $5.6 \%$ with the use of $\mathrm{LMWH}^{36}$ and up to $18 \%$ with the use of unfractionated heparin. ${ }^{42}$ While the risk of pharmacological treatment is low, it is not absent, and to justify treatment, the benefit must outweigh the risk. Currently, there are no data (in contrast to the situation for adult patients) that show a benefit for the use of anticoagulants in elective pediatric neurosurgical procedures. ${ }^{27}$

We prospectively enrolled 100 patients aged 1 month to 12 years into a study evaluating the risk of VTE in pediatric patients undergoing elective neurosurgical procedures. Ninety-one patients completed the study. Multiple factors were studied, including time in the OR. This ranged from 1:04:00 to 9:31:00 and averaged 3:42:44 \pm 1:37:51 (Fig. 2). The types of cases are shown in Table 1. There was a relatively high percentage of spine surgeries. This breakdown in case types reasonably estimated the clinical practice of the senior author (D.H.F.) at the time. None of the patients enrolled received either mechanical or pharmacological VTE prophylaxis during surgery or in the perioperative period. In addition to undergoing preoperative and post- 
operative ultrasound examinations, all patients were clinically followed for at least 1 year. No patient developed ultrasonographic or clinical evidence of VTE.

\section{Limitations}

The strength of the study is that it is prospective. The primary weakness is the relatively small number of patients, which makes the study underpowered for definitive conclusions. While we would ideally have liked to enroll more patients, we were limited by available funding.

The gold standard for diagnosis of VTE is formal venography. However, this is an invasive and therefore painful test involving the injection of contrast material. While duplex ultrasonography is not quite as reliable, we valued the ability to do a noninvasive test. Modern Doppler ultrasonography is extremely accurate in the diagnosis of DVT, with a reported sensitivity of $96 \%$ and a specificity of $98 \%-100 \% .^{18}$ Therefore, we consider the results of an ultrasound examination evaluated by an independent board-certified radiologist to be valid.

We excluded patients with a known hypercoagulable disorder, but we attempted to include any others. We only had 1 patient with a central venous line. A central line is highly correlated with VTE in the trauma literature, and thus likely to be a risk factor in elective practice. As only a single patient with a central venous line was included in our study, our ability to comment on this risk factor is minimal.

\section{Conclusions}

Governing bodies are increasingly mandating "quality metrics." In neurosurgery, prevention of VTE is a top priority. Nevertheless, instituting adult protocols for children may not be justified. Pharmacological treatment is generally safe, but not completely devoid of risk, and its efficacy in children has not been proven. In this study, we conclude that the risk of VTE in children undergoing elective neurosurgical procedures is extremely low. While the number of patients enrolled in the study precludes a determination of a definitive incidence, we can say that there were no instances of VTE in 91 patients aged 1 month to 12 years undergoing a variety of neurosurgical procedures.

We did not evaluate children with a hypercoagulable state, and we only had 1 patient with a central venous line. We would advocate consideration for treatment in these high-risk situations, but feel further study is needed.

We believe in the importance of quality initiatives and metrics, but we also believe that protocols for children must be based on study, rather than rote adaptation of adult practices. Our data suggest that the risk of VTE in children under 12 years of age is extremely low. There is a small but definable risk of bleeding with anticoagulant therapy in children. Therefore, given the low risk of developing VTE, the small risk of treatment, and the lack of data showing efficacy, pharmacological prophylaxis may not be necessary in most pediatric patients.

\section{Acknowledgments}

We would like to thank the Goodman Campbell Foundation for funding, Heather Fawcett, RN, for data management, and Dr. Boaz Karmazyn for interpretation of the duplex ultrasound studies.

\section{References}

1. Agnelli G, Piovella F, Buoncristiani P, Severi P, Pini M, D’Angelo A, et al: Enoxaparin plus compression stockings compared with compression stockings alone in the prevention of venous thromboembolism after elective neurosurgery. N Engl J Med 339:80-85, 1998

2. Allen CJ, Murray CR, Meizoso JP, Ray JJ, Neville HL, Schulman CI, et al: Risk factors for venous thromboembolism after pediatric trauma. J Pediatr Surg 51:168-171, 2016

3. Andrew M, Michelson AD, Bovill E, Leaker M, Massicotte MP: Guidelines for antithrombotic therapy in pediatric patients. J Pediatr 132:575-588, 1998

4. Baker D, Sherrod B, McGwin G Jr, Ponce B, Gilbert S: Complications and 30-day outcomes associated with venous thromboembolism in the pediatric orthopaedic surgical population. J Am Acad Orthop Surg 24:196-206, 2016

5. Beckman MG, Hooper WC, Critchley SE, Ortel TL: Venous thromboembolism: a public health concern. Am J Prev Med 38 (4 Suppl):S495-S501, 2010

6. Bernstein D, Coupey S, Schonberg SK: Pulmonary embolism in adolescents. Am J Dis Child 140:667-671, 1986

7. Bidlingmaier C, Kenet G, Kurnik K, Mathew P, Manner D, Mitchell L, et al: Safety and efficacy of low molecular weight heparins in children: a systematic review of the literature and meta-analysis of single-arm studies. Semin Thromb Hemost 37:814-825, 2011

8. Braga AJ, Young AE: Preventing venous thrombosis in critically ill children: what is the right approach? Paediatr Anaesth 21:435-440, 2011

9. Butler TJ, Sodoma LJ, Doski JJ, Cheu HW, Berg ST, Stokes GN, et al: Heparin-associated thrombocytopenia and thrombosis as the cause of a fatal thrombus on extracorporeal membrane oxygenation. J Pediatr Surg 32:768-771, 1997

10. Candrilli SD, Balkrishnan R, O'Brien SH: Effect of injury severity on the incidence and utilization-related outcomes of venous thromboembolism in pediatric trauma inpatients. Pediatr Crit Care Med 10:554-557, 2009

11. Collen JF, Jackson JL, Shorr AF, Moores LK: Prevention of venous thromboembolism in neurosurgery: a metaanalysis. Chest 134:237-249, 2008

12. Connelly CR, Laird A, Barton JS, Fischer PE, Krishnaswami $\mathrm{S}$, Schreiber MA, et al: A clinical tool for the prediction of venous thromboembolism in pediatric trauma patients. JAMA Surg 151:50-57, 2016

13. Cyr C, Michon B, Pettersen G, David M, Brossard J: Venous thromboembolism after severe injury in children. Acta Haematol 115:198-200, 2006

14. Danish SF, Burnett MG, Stein SC: Prophylaxis for deep venous thrombosis in patients with craniotomies: a review. Neurosurg Focus 17(4):E2, 2004

15. Dix D, Andrew M, Marzinotto V, Charpentier K, Bridge S, Monagle P, et al: The use of low molecular weight heparin in pediatric patients: a prospective cohort study. J Pediatr 136:439-445, 2000

16. Farray D, Carman TL, Fernandez BB Jr: The treatment and prevention of deep vein thrombosis in the preoperative management of patients who have neurologic diseases. Neurol Clin 22:423-439, 2004

17. Flinn WR, Sandager GP, Silva MB Jr, Benjamin ME, Cerullo LJ, Taylor M: Prospective surveillance for perioperative venous thrombosis. Experience in 2643 patients. Arch Surg 131:472-480, 1996

18. Gaitini D: Current approaches and controversial issues in the diagnosis of deep vein thrombosis via duplex Doppler ultrasound. J Clin Ultrasound 34:289-297, 2006 
19. Geerts WH, Bergqvist D, Pineo GF, Heit JA, Samama CM, Lassen MR, et al: Prevention of venous thromboembolism: American College of Chest Physicians Evidence-Based Clinical Practice Guidelines (8th Edition). Chest 133 (6 Suppl):381S-453S, 2008

20. Gillespie MA, Lyle CA, Goldenberg NA: Updates in pediatric venous thromboembolism. Curr Opin Hematol 22:413419,2015

21. Goldhaber SZ, Dunn K, Gerhard-Herman M, Park JK, Black PM: Low rate of venous thromboembolism after craniotomy for brain tumor using multimodality prophylaxis. Chest 122:1933-1937, 2002

22. Gonda DD, Fridley J, Ryan SL, Briceño V, Lam SK, Luerssen TG, et al: The safety and efficacy of use of low-molecularweight heparin in pediatric neurosurgical patients. J Neurosurg Pediatr 16:329-334, 2015

23. Graziano JN, Charpie JR: Thrombosis in the intensive care unit: etiology, diagnosis, management, and prevention in adults and children. Cardiol Rev 9:173-182, 2001

24. Hamilton MG, Hull RD, Pineo GF: Prophylaxis of venous thromboembolism in brain tumor patients. J Neurooncol 22:111-126, 1994

25. Hamilton MG, Hull RD, Pineo GF: Venous thromboembolism in neurosurgery and neurology patients: a review. Neurosurgery 34:280-296, 1994

26. Hamilton MG, Yee WH, Hull RD, Ghali WA: Venous thromboembolism prophylaxis in patients undergoing cranial neurosurgery: a systematic review and meta-analysis. Neurosurgery 68:571-581, 2011

27. Hanson SJ, Faustino EV, Mahajerin A, O'Brien SH, Streck CJ, Thompson AJ, et al: Recommendations for venous thromboembolism prophylaxis in pediatric trauma patients: a national, multidisciplinary consensus study. J Trauma Acute Care Surg 80:695-701, 2016

28. Harris DA, Lam S: Venous thromboembolism in the setting of pediatric traumatic brain injury. $\mathbf{J}$ Neurosurg Pediatr 13:448-455, 2014

29. Heit JA: The epidemiology of venous thromboembolism in the community. Arterioscler Thromb Vasc Biol 28:370372,2008

30. Heit JA, O'Fallon WM, Petterson TM, Lohse CM, Silverstein MD, Mohr DN, et al: Relative impact of risk factors for deep vein thrombosis and pulmonary embolism: a populationbased study. Arch Intern Med 162:1245-1248, 2002

31. Kaabachi O, Alkaissi A, Koubaa W, Aloui N, Toumi NelH: Screening for deep venous thrombosis after idiopathic scoliosis surgery in children: a pilot study. Paediatr Anaesth 20:144-149, 2010

32. Khaldi A, Helo N, Schneck MJ, Origitano TC: Venous thromboembolism: deep venous thrombosis and pulmonary embolism in a neurosurgical population. J Neurosurg 114:40-46, 2011

33. Klenner AF, Fusch C, Rakow A, Kadow I, Beyersdorff E, Eichler P, et al: Benefit and risk of heparin for maintaining peripheral venous catheters in neonates: a placebo-controlled trial. J Pediatr 143:741-745, 2003

34. Levy ML, Granville RC, Hart D, Meltzer H: Deep venous thrombosis in children and adolescents. J Neurosurg 101 (1 Suppl):32-37, 2004

35. Massicotte P, Adams M, Marzinotto V, Brooker LA, Andrew M: Low-molecular-weight heparin in pediatric patients with thrombotic disease: a dose finding study. J Pediatr 128:313318,1996

36. Massicotte P, Julian JA, Gent M, Shields K, Marzinotto V, Szechtman B, et al: An open-label randomized controlled trial of low molecular weight heparin compared to heparin and coumadin for the treatment of venous thromboembolic events in children: the REVIVE trial. Thromb Res 109:8592, 2003
37. Parasuraman S, Goldhaber SZ: Venous thromboembolism in children. Circulation 113:e12-e16, 2006

38. Punzalan RC, Hillery CA, Montgomery RR, Scott CA, Gill JC: Low-molecular-weight heparin in thrombotic disease in children and adolescents. J Pediatr Hematol Oncol 22:137142,2000

39. Raffini L, Huang YS, Witmer C, Feudtner C: Dramatic increase in venous thromboembolism in children's hospitals in the United States from 2001 to 2007. Pediatrics 124:10011008,2009

40. Ranze O, Rakow A, Ranze P, Eichler P, Greinacher A, Fusch C: Low-dose danaparoid sodium catheter flushes in an intensive care infant suffering from heparin-induced thrombocytopenia. Pediatr Crit Care Med 2:175-177, 2001

41. Raskob GE, Silverstein R, Bratzler DW, Heit JA, White RH: Surveillance for deep vein thrombosis and pulmonary embolism: recommendations from a national workshop. Am J Prev Med 38 (4 Suppl):S502-S509, 2010

42. Revel-Vilk S, Chan AK: Anticoagulation therapy in children. Semin Thromb Hemost 29:425-432, 2003

43. Rohrer MJ, Cutler BS, MacDougall E, Herrmann JB, Anderson FA Jr, Wheeler HB: A prospective study of the incidence of deep venous thrombosis in hospitalized children. J Vasc Surg 24:46-50, 1996

44. Setty BA, O'Brien SH, Kerlin BA: Pediatric venous thromboembolism in the United States: a tertiary care complication of chronic diseases. Pediatr Blood Cancer 59:258-264, 2012

45. Spentzouris G, Scriven RJ, Lee TK, Labropoulos N: Pediatric venous thromboembolism in relation to adults. J Vasc Surg 55:1785-1793, 2012

46. Tabori U, Beni-Adani L, Dvir R, Burstein Y, Feldman Z, Pessach I, et al: Risk of venous thromboembolism in pediatric patients with brain tumors. Pediatr Blood Cancer 43:633636, 2004

47. Van Arendonk KJ, Schneider EB, Haider AH, Colombani PM, Stewart FD, Haut ER: Venous thromboembolism after trauma: when do children become adults? JAMA Surg 148:1123-1130, 2013

48. Vavilala MS, Nathens AB, Jurkovich GJ, Mackenzie E, Rivara FP: Risk factors for venous thromboembolism in pediatric trauma. J Trauma 52:922-927, 2002

49. Yen J, Van Arendonk KJ, Streiff MB, McNamara L, Stewart FD, Conner KG, et al: Risk factors for venous thromboembolism in pediatric trauma patients and validation of a novel scoring system: the risk of clots in kids with trauma score. Pediatr Crit Care Med 17:391-399, 2016

\section{Disclosures}

The authors report no conflict of interest concerning the materials or methods used in this study or the findings specified in this paper.

\section{Author Contributions}

Conception and design: Fulkerson. Acquisition of data: all authors. Analysis and interpretation of data: Fulkerson. Drafting the article: Fulkerson, Scherer, White, Shaikh. Critically revising the article: Fulkerson. Reviewed submitted version of manuscript: Fulkerson. Approved the final version of the manuscript on behalf of all authors: Fulkerson. Administrative/technical/material support: Fulkerson. Study supervision: Fulkerson.

\section{Correspondence}

Daniel H. Fulkerson, Indiana University School of Medicine/ Goodman Campbell Brain and Spine, Riley Hospital for Children, 702 Barnhill Dr. \#1134, Indianapolis, IN 46202-5200. email: dfulkers@iupui.edu. 